\title{
Some Natural Remediates for Restoration of Contaminated Soil
}

\author{
${ }^{1 *}$ S. Moin and ${ }^{2}$ N. Emad \\ 1*Department of Botany, Federal Urdu University of Arts, Sciences \& Technology Karachi, \\ ${ }^{2}$ Department of Botany, University of Karachi, Pakistan, \\ *Corresponding Author Email: sumeiramoin@ hotmail.com
}

\begin{abstract}
:
The soil, an essential part of this universe, comprises elements, compounds, and a mixture responsible for sustainable life on the earth's crust. Nowadays, under the threat of modernization and urbanization, the composition of the soil is altered. Soil contamination and depletion are the gift of the modern world where soil pollution results from the management of solid and liquid hazardous waste into it, resulting in alteration in its composition, consequently; poor-quality fruits and vegetables available for human consumption. This review article discusses some natural remediate like AM fungi, marine green algae, and used tea wastes to restore the soil. The application of these natural materials proves to be a suitable replacement of any chemical fertilizer, applied to improve the soil quality with no further harm to soil followed by a large and good quantity of crops. These natural remediate help in checking the movement of heavy metals from soil to plants root and act as the best cost-effective technology with the replacement of any standard chemical fertilizer, where only transportation is required to add these materials for soil restoration healthy growth of the plants.
\end{abstract}

Keywords: Bioremediation, Soil, Metals, VAM, Tea waste, Marine alga

\section{INTRODUCTION:}

The world's current advancement appears as detrimental to the soil, especially for that soil where crops were cultivated. This advancement ignored the proper placement of the discharged material, especially related to the inorganic metals and organic compounds, resulting in harmful effects on soil composition ${ }^{1}$. The soil pollution-induced oxidative stress, which is generated due to biotic and abiotic stress, results in alteration of entire metabolic activities of the plant to overcome it ${ }^{2}$. The oxidative species like singlet oxygen and hydroxyl radical are powerful oxidizing agents, toxic to the plant to which plants metabolism modified for survival in contaminated soil through high proline, polyphenols, or carotenoids content ${ }^{3}$. Currently, searches are based on remediation technologies for soil conditioning using natural material to check the metal movement in plant roots ${ }^{4}$. There are several types of natural remediates with their different types used according to the soil's needs like VAM fungi, microbes and bacteria, fresh water and marine algae, biochar, and other household waste, including tea waste ${ }^{5-}$ 12

This review article will discuss the soil remediation by three natural remediates near plant growers for healthy crop cultivation. The pot experiment conducted and discussed using Sterilized seeds followed by the control plants, metalcontaminated plants ( $\mathrm{Hg}, \mathrm{Cd}$ and $\mathrm{Cr}$ ), VAM inoculated, tea waste treatment, and application of marine alga in diverse sets of triplicates with different concentration and different plants species for validation of bioremediation under different conditions like drought, and temperature. Initially, the growth rate is monitored as a first remediation effect on contaminated soil and compared with their Control respective sets. The other metabolic parameters were monitored using advanced technologies like SEM, UV/Visible, HPLC etc ${ }^{8-12}$.

\subsection{Mycorrhizae Fungi As A Natural Resource:}

Mycorrhizae are naturally available fungi present in most soil and easily separated by using multiple sieves used for bioremediation of the soil. The beneficial relationship of the two partners of the plant kingdom is defined as the symbiosis developed in soil and plant roots for developments of both plants and Mycorrhizae where AM fungi propagate inside plants roots through their mycelium or sometimes on the surface of the roots. Both partners help each other so that fungus helps provide nutrients and water to the roots from soil, and plants provide carbon $(\mathbf{C})$ to fungi to develop their hyphal network system. The process of exchange of nutrient and $\mathbf{C}$ consequently activate the plants' photosynthetic activity, which overall results in stimulated metabolic pathway of the plants followed by healthy growth. In some cases, the beneficiary part of dual symbiosis is hidden, and the relationship seems to be harmful. Still, this factor is mainly related to soil conditions that sort out after analysis of the soil and then introduce different natural remediates results in soil condition with better growth 
of the plants. It is also important to note that when soil conditions are good enough for growth, there is no need of any chemical and biological remediates ${ }^{13}$.

There are different types of Mycorrhizae are found in soil naturally which connect to the plants in two ways like i) surrounds the surface of the roots referred to as ectomycorrhiza, found 5 to $10 \%$ of the terrestrial plant while ii) fungi penetrates in the roots called endomycorrhiza, or one can say that their hyphae network congested in between the cell wall and the cell membranes of the roots found in $80 \%$ of the plant's species with beneficiary effects in the form of nutrient supply ${ }^{14}$. This fungal form develops its hyphal network and provides nutrients to the plants in stress conditions for better development. It receives $\mathbf{C}$ from the plant's carbohydrates, thereby increasing its rate of photosynthesis. The significant nutrients (nitrogen, phosphorous, potassium, calcium, sulfur, and zinc) from the soil provided by VAM fungi include some specific mechanism which operated in the soil due to the presence of spore and process of solubilization, helps them to move inside in the roots ${ }^{13}$.

Taxonomically, Arbuscular mycorrhizae "AM" (formerly VAM) fungi are famous for its association with the roots of plants related to phosphorus ability and effective Control during drought stress and the ability for nutrient uptake. They form arbuscules, which are the exchange sites for nutrients such as phosphorus, carbon, and water. Vesicular-arbuscular mycorrhiza (VAM) is formed by the symbiotic association between certain phycomycetous fungi and angiosperm roots ${ }^{15}$. In other words, the fungi cannot grow in the absence of their plant host ${ }^{13}$. The fungal specialty is dominant in drought stress. Its function is dominant in the modified mechanism of chemical transformation of proline in releasing a water molecule coupled with minimization of the oxidative stress or conversion of singlet oxygen species into triplet oxygen species $^{12}$.

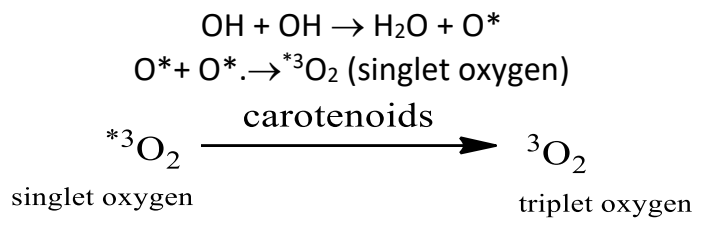

1.1- Remediation of singlet oxygen species through Proline and Carotenoids Quenching Action under biotic and biotic stress

$$
\begin{gathered}
{ }^{1} \mathrm{C}+\mathrm{hv} \rightarrow{ }^{1} \mathrm{C}^{*} \quad \text { (photochemical excitation) } \\
{ }^{1} \mathrm{C}^{*} \rightarrow{ }^{3} \mathrm{C} \quad \text { (inter-system crossing) } \\
{ }^{3} \mathrm{C}+{ }^{3} \mathrm{O}_{2} \rightarrow{ }^{1} \mathrm{C}+{ }^{1} \mathrm{O}_{2} \text { (photo-sensitization) } \\
{\left[{ }^{1} \mathrm{O}_{2}+\mathrm{A}\right] \leftrightarrow{ }^{1}\left[\mathrm{O}_{2}^{\delta^{-}} \leftarrow \mathrm{A}^{\delta^{+}}\right] \text {(reversible formation of charge transfer compound) }} \\
{ }^{1}\left[\mathrm{O}_{2}{ }^{\delta^{-}} \leftarrow \mathrm{A}^{\delta}\right] \rightarrow{ }^{1}\left[\mathrm{O}_{2}^{\delta^{-}} \leftarrow \mathrm{A}^{\delta^{+}}\right] \rightarrow{ }^{3} \mathrm{O}_{2}+\mathrm{A} \text { (Physical quenching) } \\
{ }^{1}\left[\mathrm{O}_{2}{ }^{\delta^{-}} \leftarrow \mathrm{A}^{\delta^{+}}\right] \rightarrow \text { product (Chemical Quenching) }
\end{gathered}
$$

\subsubsection{Important function of AM Fungi:}

There are two crucial functions of AM fungi during dual symbiosis in drought dress and metal contamination, like increase $\mathbf{P}$ contents and photosynthetic activity. Phosphorus is abundant in soil, and essential element, but availability to plant is low due to mineral transformation into phosphates. Nevertheless, it is an essential element for the growth of the plant, including cell development and flower development, to produce seeds, as phosphorus counts as an energy provider to the plants. Results reported in Table 1 showed the $\mathbf{P}$ contents in different trails of the plant, Like Control, plant, Mycorrhizal plant (MP), Drought Mycorrhizal plant (DMP), and Carbon extreme condition Mycorrhizal plant (CMP). It wass observed that Mycorrhizal plants in different condition reflect enhanced contents of $\mathbf{P}$. Therefore, it is established that Arbuscular Mycorrhizal (VAM) is a fungus which can help to solubilize the phosphates available in soil abundantly. AM offers the required power to defy diseases, bacteria, and adverse climate conditions ${ }^{16}$.

Table 1: The availability of Phosphorus content (ppm) in different condition to the Conocarpus erectus L. plant.

\begin{tabular}{cccccc}
\hline Sample code & Soil & Roots & Stems & Leaves & $\begin{array}{c}\text { Significance (p) } \\
\mathrm{P}<0.001\end{array}$ \\
\hline Standard & $6.82 \pm 0.095$ & - & - & - & $0.000 * * *$ \\
Control plant & $3.50 \pm 0.040$ & $3.40 \pm 0.060$ & $5.5 \pm 0.1$ & $4.30 \pm 0.047$ & $0.000 * * *$ \\
MP & $4.61 \pm 0.159$ & $3.40 \pm 0.025$ & $5.80 \pm 0.040$ & $5.22 \pm 0.064$ & $0.000 * * *$ \\
DMP & $6.71 \pm 0.055$ & $3.9 \pm 0.045$ & $6.21 \pm 0.090$ & $5.91 \pm 0.055$ & $0.000^{* * *}$ \\
CMP & $4.72 \pm 0.064$ & $3.60 \pm 0.040$ & $5.61 \pm 0.036$ & $5.30 \pm 0.047$ & $0.000^{* * *}$ \\
\hline
\end{tabular}

The role of VAM fungi in duel symbiosis is fundamentally related to the two basic elements. These are $\mathbf{C}$ and $\mathbf{P}$, where $\mathbf{C}$ is used by VAM fungi for the development of its hyphal network and $\mathbf{P}$ for speed up the ATP function in 
photosystem II. It was established that VAM fungi rely on freshly prepared carbon of starch, arranged during active photosynthesis (Figure 1) as it is already reported by numerous researchers that during duel symbiosis, the rate of photosynthesis increases. Moreover, it was also suggested that AM fungi take $\mathbf{C}$ from plants due to which $\mathbf{C}$ assimilation in plant increases or rapid photoactivity observe due to larger surface area of leaves where leaves absorb more $\mathrm{CO}_{2}$ owing to the high availability of $\mathrm{P}$ contents in Conocarpus erectus $L$ plants ${ }^{8,10-12}$

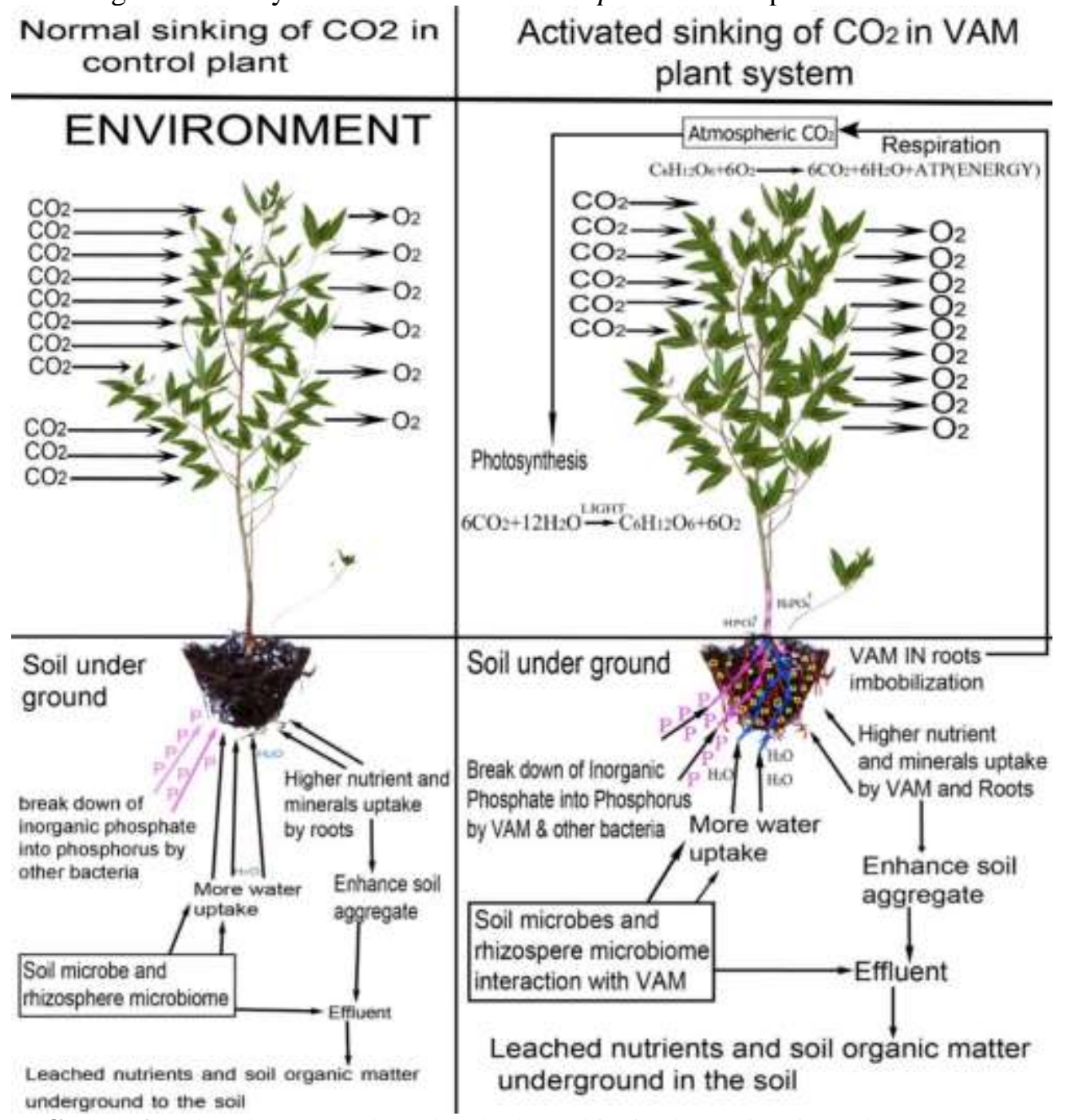

FIGURE 1. The plant growth under dual symbiosis showed activated photosystem and growth rate ${ }^{8}$

Experimental evidence regarding $\mathbf{P}$ accumulation under VAM inoculation assigned a new role of Fungi as a bio-activator and bio-convertor of insoluble $\mathbf{P}$ to soluble where higher $\mathbf{P}$ contents and photosynthesis rate observed. It is believed on experimental evidence and literature search that the $\mathbf{P}$ contents of the soil solubilized into the anionic form $\mathbf{H}_{2} \mathbf{P O}_{4}^{-}$which is very usable by the plants.

$$
\text { Organic/inorganic component }+ \text { solubilizing agent } \stackrel{\text { Rhizosphere }}{\text { VAM }}-\mathrm{H}_{2} \mathrm{PO}_{4}^{-}
$$

\subsubsection{Remediation of metal contaminated soil by VAM:}

The ecosystem efficiency of the soil is markedly increasing in the presence of the AM fungi, especially in the presence of the contaminants and drought stress where AM fungi act as a remediator followed by important parameters in relation to the growth of the plant, including i) enhanced plant water and nutrient uptake ii) improvement in soil quality, iii) enhanced interaction to other microbes, iv) effective check on soil pathogens, and v) inhibit metal accumulation in roots ${ }^{17}$.

Heavy metals are an important part of human life, but on the other hand, it acts as a source of pollution, consequences of anthropogenic activities. Plant growth is markedly affected by the presence of the heavy metal as it accumulates in roots with other nutrient ions, generated false signals, consequently the superiority of nutrition affected. The deleterious effects 
of heavy metals on soil and roots can be determined through carbonate concentration, roots exudates as well as organic matter, microbes, clay, and $\mathrm{pH}$. The presence of microbial life with organic matter help in the immobilization of heavy metals Like $\mathbf{C u}, \mathbf{C d}$, etc., while roots exudates such as some organic acid increase the mobility of the metal through soluble complex formation. It was established that AM fungi able to remediate heavy metal stress on the growth of plants in comparison of non-treated plants and lesser amount of metal reported in roots of AM inoculated T. praecox plants, belongs the Brassicaseae family, under duel symbiosis followed by the high tolerating ability of metal stress in T. praecox ${ }^{17}$.

\subsection{Function Of Tea Waste In Soil Remediation:}

Several kinds of research pertain to soil pollution that metal toxicity is injuriousness, especially related to $\mathbf{P b}$ and $\mathbf{C d}$ metals with no biological function plants ${ }^{18}$. Wasted black tea (WBT) is a low-cost sorbent observed, effective in the removal of heavy metals. The remediation of metals by black tea waste is found to be the best eco-friendly technique as it has a relatively high adsorption capacity for heavy metals ${ }^{19}$ wherever $\mathrm{pH}$ is important in the adsorption of heavy metals as the level of $\mathrm{pH}$ allows maximum adsorption of heavy metal ${ }^{19}$. Tea waste can adsorb the maximum concentration of metal due to high porosity and availability of functional groups on its surface ${ }^{20,21}$. Numerous methods and technologies are available for the removal of heavy metals from the soil, but bioremediation of heavy metals through tea waste is a green technology that showed no harm to the soil. In any technology cost to remove or remediate the toxicant is significant, whereas tea waste costs only transportation charges for collection of waste from far and near places followed by very simple application on soil $^{22}$.

Tea, a most used drink for gaining a fresh and active look by humans as it is an alcohol-free and caffeinated beverage in the world, prepared from the fresh leaves of the tea plants through boiling ${ }^{23}$. Generally, it is very beneficial in the form of green tea leaves for human, and its waste applied as a fertilizer as it consists of $\mathbf{K}, \mathbf{N}$, and $\mathbf{P}$, which recommended to tea waste as natural available NPK bio-fertilizer or eco-friendly green fertilizer to enhance the soil conditioning for good and safe growth of the plants. The function of the tea- waste in soil conditioning to manage the soil moisture and oxygen level for soil microbial life, especially to earthworms' survival followed by the prevent pathogenic effect on plants ${ }^{24}$.

\subsection{Bioremediation Through Tea Waste:}

Biosorption or bioremediation of metal in the soil through green plant wastes or direct plant use related with the absorption or bond formation. It involves complex formation with the particular group of compound present in it, termed as ion exchange, physical linkage or adsorption or chelation, or found a place in between structural polysaccharides ${ }^{25}$. The biosorption mechanism of metal on tea waste leaves is based on the group of bioactive compounds like phenols, the hydroxyl group of compounds like polysaccharides, carboxylate, and oxyl groups. These groups of compounds are responsible for their ion exchange or chelation with metal, followed by a complex formation which checked the movement of the metal and blocked its accumulation in roots ${ }^{26}$.

It is to remember that heavy metals are naturally occurring parts of the soil which is not toxic to the soil or human through the food chain while toxic when disposed of under anthropogenic activities. Recently few methods based on available natural material were developed, which showed an effective control of the metal within the soil. These metals cannot be decomposed or destroyed but can control through complex formation or by changing their oxidation states. As a result of the alteration of its oxidation state, the metal may become either: (i) more water-soluble and is removed by leaching, (ii) inherently less toxic, (iii) less water-soluble so that it precipitates and then becomes less bioavailable or removed from the contaminated site, or (iv) volatilized and removed from the polluted area $^{25}$.

\subsection{Remediation Of Cd Metal Within Soil:}

Soil contaminated with the metal showed an adverse effect on plant metabolism, which can observe clearly through its growth or morphological appearance. A pot experiment conducted for soil remediation in the presence of different percentages of the $\mathbf{C d}$ while remediation performed through tea waste application, Investigation of $\mathbf{C d}$ toxicity on leaves appeared as reduced surface area $(87 \pm 0.5)$ when treated $400 \mathrm{ppm}$ while recovered under tea waste application over control plant (Figure 2). It was initially related to the generation of reactive oxygen species, which causes oxidative burst followed by variations in the usual metabolic pathway of plants. The oxidative stress results in a low growth rate reflected by reduced photosynthesis and transpiration rate, which were linked with a reduced surface area of the leaves. When plants treated with tea leaves waste in the presence of $\mathbf{C d}$ metal, an enhanced growth rate was observed, where high growth rate and large surface indicate the absence of the $\mathbf{C d}$ metal and soil remediation ${ }^{27}$. 


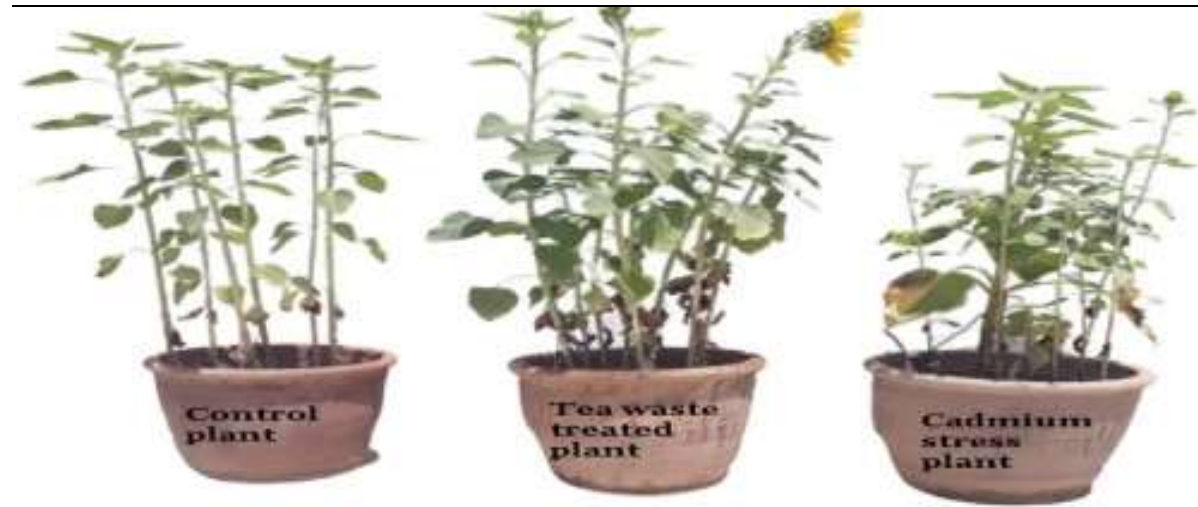

FIGURE 2: The remediation of Cd contaminated soil by Tea waste

$\mathbf{H}_{2} \mathbf{O}_{2}$ is a significant molecule of plant metabolism where oxygen is release through stomata, while in the presence of heavy metal, reduced leaves size with reduced stomata inhibits the liberation of oxygen followed by oxidative damage in plants. The Cd treated plant with tea waste showed larger surface, and good growth rate which showed that tea waste effectively controls the $\mathbf{C d}$ and control $\mathbf{R O S}$ like $\mathbf{H}_{\mathbf{2}} \mathbf{O}_{\mathbf{2}}$ and which is vital compound and a signaling molecule followed the regular metabolic pathway for normal numerous metabolic process of the plants like translocation, photosynthesis, respiration, and transpiration. Therefore, these sequences enhanced the crop yield. Presently $\mathbf{H}_{2} \mathrm{O}_{2}$ plays a role as a signaling molecule in signal transduction pathways important to acclimation and defensive against abiotic stresses ${ }^{28}$. Mixing of the tea waste in the polluted soil results in normal seed germination, photosynthetic rate, amino acid and proteins, carbohydrate formation, and inhibit automatic cell death due to the presence of $\mathbf{C d}$, followed by flowering and root system development. $\mathbf{H}_{2} \mathbf{O}_{2}$ is the utmost steady, sustainable compound manufactured in plant cells during respiration and photosynthesis. These outcomes suggest metal pollution activate the production of $\mathbf{H}_{2} \mathbf{O}_{2}$ in cellular transduction signaling pathways and dissociated to ${ }^{*} \mathrm{OH}$ radical, which is power full oxidizing species

$$
\begin{gathered}
2 \mathrm{O}_{2}^{-*}+2 \mathrm{H}^{+} \rightarrow \mathrm{H}_{2} \mathrm{O}_{2}+\mathrm{O} \\
\mathrm{H}_{2} \mathrm{O}_{2} \rightarrow{ }^{*} \mathrm{OH}+{ }^{*} \mathrm{OH} \text { in presence of } \mathbf{C d}
\end{gathered}
$$

\subsection{Marine Algae As Bioremedator:}

Metal pollution is the gift of the industrial revolution and now part of life, followed by its toxic effects on soil and accumulation in crops and vegetables. Moreover, it is acquiring the status of the part of the food and needs to be addressed. Bioremediation through biosorption using non-regular and low-cost constituents has gained attention in the last few decades, as it extends an inexpensive and effective substitute to the already established technologies.

Several experiments at laboratory scales showed that the marine biomass proves to be efficient in concentrating inorganic metal ions on their surface. The biosorption capability of the marine green seaweeds for $\mathbf{H g}$ relies on their cell wall chemical constituents which are mainly composed of the alginates and fucoidans with several functional groups that interact with metal ions and checked their mobility in the roots of plants ${ }^{29}$.

Marine seaweeds are considered renewable, inexpensive biological resources. That helps in protecting the crops safe from the interaction of heavy metals. There are several uses of seaweeds, including extraction of biofuel to extraction of some important natural products. However, the use of seaweeds as biomass or surface in bioremediation for treatment of soil or wastewater gaining attention from researchers to control heavy metal pollution for the safety of food is important ${ }^{7}$. 

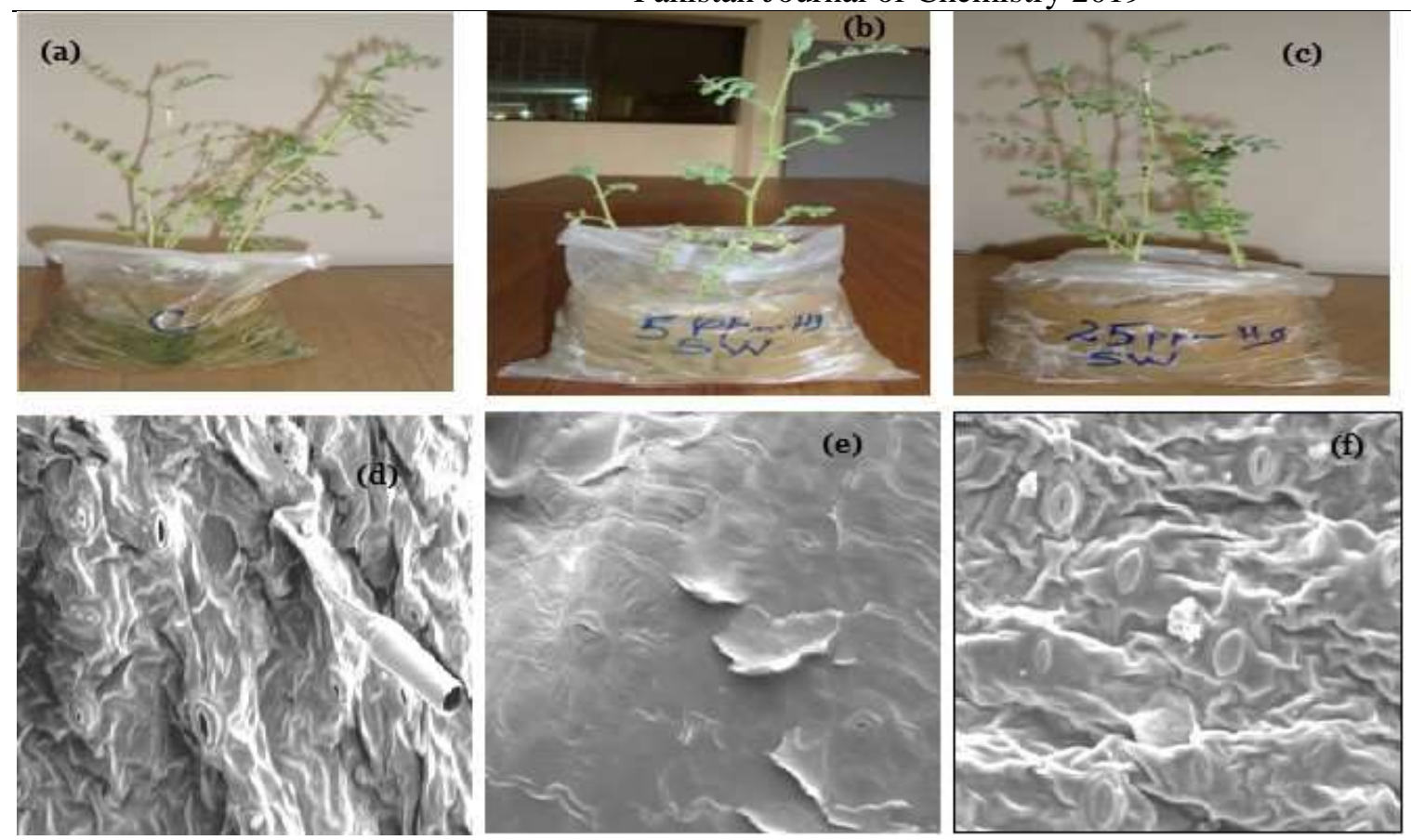

FIGURE 3: Remediation of $\mathrm{Hg}$ through green Seaweeds a) control plant, b) plant grown in Cd contaminated soil, c) remediation of contaminated soil by Seaweeds, d) size of stomata of control plant, e) size of stomata of contaminated plant f) size of stomata in remediated soil

The investigation of bioremediation of $\mathbf{H g}$ in the soil through marine algae showed that it successfully checks the mobility of the metal from root to shoot, and the plant observed good growth under contaminated soil. This indicated the biosorption of $\mathbf{H g}$ takes place on the surface of the Codium iyengrii. The surface morphology of the leaves through scanning electron microscopy (SEM) was conducted to validate the process of photosynthesis (under metal toxicity), which was the initial stage of growth after seed germination. It was observed that $\mathbf{H g}$ contaminated plant showed the rough surface of the leaves with a very reduced stomatal opening. It was a significant point of Carbon dioxide sinking in comparison to control plants (Figure 3), while plants grown with seaweeds treatment showed a normal growth rate. Moreover, SEM analysis showed that $\mathbf{H g}$ triggered morphological worsening followed by stunt stomatal opening with a smaller number of stomata (Figure 3). Hg accumulation exhibit stress conditions in plant that display xeromorphy. The obstructed and immersed stomata is an example of the xeromorphic character; observed in SEM analysis during investigation ${ }^{30}$.

Stomata are the openings surrounded by two guard cells, located between the epidermis of leaves to conduct two basic functions i) release of water vapors produced during transpiration while ii) absorption of $\mathbf{C O}_{2}$ with the release of oxygen required for photosynthesis. Reduced number of stomata in leaves under $\mathbf{H g}$ accumulation indicates the inhibition of both processes, consequently growth of the plant affected. Hg stress mainly puts an impact on osmotic potential and decreases water transportation from distorted roots, cause a reduction in the number of stomata followed by a reduction in transpiration $^{30}$. Reduced transpiration decreased the availability of the solutes to a sustainable metabolic pathway, while the addition of powder of marine green algae codium iyengrii remediate the epidermal structure, which results in good growth of the plants. The rough structure of leaves also influences photosynthesis ${ }^{9}$.

\subsection{Growth Rate Of The Plants Under Bioremediation:}

The remediation of soil in the presence of $\mathbf{H g}$ on cicer arietinum showed that dry marine green algae Codium Iyengrii showed great effect where healthy growth of the plants results in the presence of $\mathbf{H g}$ indicate that movement of metal completely checked and probably metal adsorbed on the surface of the algae. Following effects of addition of marine alga were observed on cicer arietinum under $\mathbf{H g}$ contamination ${ }^{30}$.

i) Improvement in soil structure

ii) Healthy growth of the cicer arietinum

iii) Repairing of the epidermal layer of the leaves

iv) Activated transpiration and photosynthesis

v) The regular morphological and physiological process 


\section{CONCLUSION}

Soil contamination with metal showed deleterious effects on growth rate due to which metabolic pathway is altered. The remediation processes involve natural, cost-effective material, prove an excellent remediator to control the movement of the metal ions as practically each treated plant under various metals noxiousness showed approximately the same growth rate when compared with non-contaminated plants.

\section{ACKNOWLEDGMENT:}

The Corresponding Author is thankful HEC Pakistan for providing financial assistance through Project No. 212077/SRGP/R\&D/HEC/2018.

\section{REFERENCES:}

1. Lee, K. E., \& Pankhurst, C. E. (1992). Soil Research, 30(6), 855-892.

2. Lee, J. C., Son, Y. O., Pratheeshkumar, P., Shi, X. Free Radical Biol. Med., (2012), 53, 742-757.

3. Shahid, M., Dumat, C., Silvestre, J., Pinelli, E. Biol. Fertil. Soils, (2012), 48, 689-697..

4. Gadepalle, V. P., Ouki, S. K., Herwijnen, R. V., \& Hutchings, T. (2007). Soil \& Sediment Contamination, 16(2), 233-251.

5. Fourest, E., Roux, J. C. Appl. Microbiol. Biotechnol., (1992), 37, 399-403..

6. Askari, S., Arif, N., Naheed, S. J. Pharma. Phytochem. (2017), 6, 1807-11.

7. Askari, S., Uddin, F., Azmat, R. Pak. J. Bot., (2007), 39, 1089.

8. Peng, W., Li, X., Xiao, S., \& Fan, W. (2018). Journal of soils and sediments, 18(4), 1701-1719.

9. Askari, S., Azmat, R. J. Pharmacogn. Phytochem., (2016), 170.

10. Azmat, R., Hamid, N., Moin, S. Recent Pat. Biotechnol., (2015), 9, 63-74.

11. Azmat, R., Hamid, N., Moin, S., Saleem, A. Recent Pat. Biotechnol., (2015), 9, 130-138.

12. Azmat, R., Moin, S., Saleem, A., Hamid, N., Khursheed, A., Ahmed, W. Recent Pat. Biotechnol., (2018), 12(1), 65-76.

13. Biologydictionary.net Editors. "Mycorrhizae." Biology Dictionary, Biologydictionary.net, 18 May. (2017), https://biologydictionary.net/mycorrhizae/

14. Untamedscience.com Lindsay Van Someren "Mycorrhizae". Published on: 07/ (2016), https://untamedscience.com/biology/ecology/mycorrhizae/

15. Sullia, S. B. In Horticulture-New Technologies and Applications (1991), (pp. 49-53). Springer, Dordrecht.

16. Mathrubhumi.com "Agriculture" Role of VAM in Increasing Yield. Published November 22, 2015 https://english.mathrubhumi.com/agriculture/role-of-vam-in-increasing-yield-english-news-1.688291

17. Miransari, M. Biotechnol. Adv., (2011), 29, 645-653.

18. Ziarati, P., Zolfaghari, M., Azadi, B. Int. J. Plant, Anim. Environ. Sci., (2014), 4, 479-486.

19. Mohammed, R. R. Arabian J. Sci. Eng., (2012), 37, 1505-1520.

20. Malakahmad, A., Tan, S., Yavari, S. Journal of Chemistry, (2016).

21. Amarasinghe, B. M. W. P. K., Williams, R. A. Chemical Engineering Journal, (2007), 132, 299-309.

22. Thakur, L. S., Parmar, M. Int. J. Chem. Phys. Sci., (2013), 2, 6-19.

23. Zhang, J., Yang, R., Chen, R., Peng, Y., Wen, X., Gao, L. Int. J. Environ. Res. Public Health, (2018), $15(1), 133$.

24. Henri.nestle.com Fahad Muhammad "Using Tea Waste As Fertiliser" (2018) https://henri.nestle.com/liveprojects/everyday-evergreen-utilising-wasted-tea-

fertiliser\#: :text=Looking\%20at\%20the\%20research\%2C\%20we,even\%20allows\%20earthworms\%20to\%20thriv e.

25. Kumar, B., Smita, K., Flores, L. C. Arabian J. Chem., (2017), 10, S2335-S2342.

26. Hussain, S., Anjali, K. P., Hassan, S. T., Dwivedi, P. B. Appl. Water Sci., (2018), 8, 1-16.

27. Azmat, R. Integr. Waste Manage., (2011), Volume II, 1149.

28. Zentgraf, U., Zimmermann, P., Smykowski, A. In Senescence. IntechOpen, (2012), 31-50.

29. Kumar, J. N., Oommen, C., Kumar, R. N. American-Eurasian J. Agric. \& Environ. Sci, (2009), 6, 317-323.

30. Askari, S., Azmat, R. Pak. J. Bot, (2013), 45, 1721-7. 